\title{
Adaptive surrogate model-based fast path planning for spacecraft formation reconfiguration on libration point orbits
}

\section{Haijun Peng and Wei Wang}

\begin{abstract}
This work considers the optimization of fast path planning for spacecraft formation reconfiguration on libration point orbits. An adaptive surrogate model is proposed for solving the above optimization problem in abnormal conditions and/or emergency cases. First, a multi-layer optimization problem is established. In the outer loop of the multi-layer optimization, the nonlinear optimal control problem, taken as a dynamic optimization, is solved to obtain the optimal transfer paths of all spacecraft in the formation. In the inner loop of the multi-layer optimization, the parameter optimization problem, taken as a static optimization, is solved to obtain the states of all the spacecraft within the maximized area of the spacecraft formation. Then, an adaptive surrogate model is proposed. Because the multi-layer optimization problem is typically time-consuming and computationally expensive, the adaptive surrogate model is constructed using data drawn from the multi-layer optimization model and provides a fast approximation of the objective at new design points. Therefore, the adaptive surrogate model is feasible and cheap for spacecraft formation reconfiguration. Finally, numerical simulations show that the adaptive surrogate-based parameter optimization has advantages over the direct global optimization method. The accuracy, efficiency and robustness of the proposed adaptive surrogate model have also been compared between the different types of surrogate models.
\end{abstract}

\section{Keyword}

Spacecraft formation reconfiguration, Fast path planning, Adaptive surrogate model, Libration point orbit, Circular restricted three-body problem.

Haijun Peng (Corresponding author)

Department of Engineering Mechanics, State Key Laboratory of Structural Analysis for Industrial Equipment, Dalian University of Technology, Dalian, 116024, China

e-mail: hipeng@dlut.edu.cn 


\section{Introduction}

The exploration of deep space, especially of a libration point space environment, is an important research topic that has attracted much attention in recent years [1-10]. These positions are ideal for searching for habitable terrestrial exoplanets or situating a set of interferometer space telescopes or midway refueling space stations for large missions. Deep space formation flying technology is essential to meet the increasing demand for deep space exploration. Thus far, both spacecraft formation keeping and reconfiguration, potential applications that involve spacecraft formations flying on the libration points, have attracted great interest for formation control strategies in a multibody regime.

Various strategies and approaches have been proposed for the reconfiguration of the spacecraft formation on libration point orbits. Reference [5] gives comparisons among the optimal nonlinear control, geometric control, linear quadratic regulators, and input state feedback linearization. Reference [6] proposes a finite element method for solving for optimal control and applies it to spacecraft formation reconfiguration in a libration point regime of the sun-earth system. Reference [7] develops a technique based on generating functions for designing spacecraft formation reconfiguration in Hill three-body dynamics. Reference [8] presents an efficient parameter optimization algorithm for collision-free energy sub-optimal path planning of spacecraft flying in formation in deep space. Based on the characterization of requirements and constraints, different algorithms for centralized optimal formation planning and coordination have been developed in reference [9]. Reference [10] employs a finite-dimensional parameter optimization method for spacecraft formation reconfiguration guidance.

Although the literature cited above has emphasized the problem of spacecraft formation reconfiguration near libration point orbits, particularly in path planning, avoiding collisions and balancing energy, etc., all the reconfiguration missions are planned based on known information in normal circumstances and can be evaluated off-line before the actual implementation of the spacecraft formation reconfiguration mission. However, an engine failure or other mechanical defect on a certain spacecraft in the formation will arise in abnormal conditions and/or emergency cases. In these extreme cases, all the information for the spacecraft cannot be obtained in advance, and primary fast path planning for spacecraft formation reconfiguration is particularly important for the follow-up normal missions. Therefore, the development of fast path planning for spacecraft formation reconfiguration in case of emergency is an active area of research.

From the point of view of dynamics and control, there are two primary scientific problems to be 
solved before the actual implementation of spacecraft formation reconfiguration: (1) the scientific objective of the spacecraft formation; i.e., the new form taken by the spacecraft formation, based on the present state and number of spacecraft; (2) with the new form taken by the spacecraft formation known, the path planning for the spacecraft formation reconfiguration. Regarding the first scientific problem, the parameter optimization method is usually employed to plan the new geometric configurations and to obtain the new states of the spacecraft in their new formation. In the second scientific problem, the nonlinear optimal control method is always used to design the optimal transfer paths of all spacecraft from the failure formation to the new formation. It is essentially a coupling system between the first and the second scientific problems, and neither of the above problems can be solved alone. This leads to complex nonlinear optimization problems, in which computational efficiency is a determining factor for successful implementation of spacecraft formation reconfiguration in extreme cases.

Gradient-based methods and intelligent algorithms are usually employed to solve the above complex nonlinear optimizations problems. However, the gradient-based methods have a high efficiency but will obtain locally optimal results [11]. Moreover, the optimization problem proposed in this paper has strongly implicit relationship between the design variables and the objective function, so the analytic gradient information cannot be obtained from the above complex nonlinear optimization. The intelligent algorithms are convenient to implement and can obtain globally optimal results. However, they require thousands of function evaluations [12], and this process may require unacceptable computational cost.

Motivated by the requirements for high computational efficiency for spacecraft formation reconfiguration in extreme cases, an efficient adaptive surrogate model-based fast path planning strategy for spacecraft formation reconfiguration on libration orbits will be investigated in this paper. Surrogate-based optimization (SBO) is an important and efficient way to quickly access the optimal results [13]. Because the surrogate model uses an explicit and approximate expression to replace the true complex model, the optimization based on the surrogate model has a very high efficiency. As a result, SBO has been shown to be an effective approach for the design of computationally expensive models, such as many optimizations in aerospace systems [14-16]. In this paper, for the first scientific problem, the present geometric configuration of spacecraft can be measured in abnormal conditions and/or emergency cases. The desired new geometric configuration of spacecraft should be designed according to the requirement of different space mission. One requirement of these different space 
mission is to obtain high-resolution for deep space explorations and studies of outer space. In particular, a larger area that is surrounded by the spacecraft formation can obtain a higher resolution. Thus, maximum area of the spacecraft formation under some geometric constraint should be a key objective. For the second scientific problem, the fuel consumption should be minimized in path planning for spacecraft formation reconfiguration. Sufficient remaining fuel is a prerequisite for the follow-up normal missions. Based on the above analysis, a multi-layer optimization model is first formalized for the fast spacecraft formation reconfiguration problem. Then, the adaptive surrogate model is proposed for fast solving of the above multi-layer optimization problem. Finally, the numerical simulations are implemented to test the effectiveness of the proposed fast path planning strategy for the spacecraft formation reconfiguration problems.

The paper is organized as follows: in section 2 , a brief review of spacecraft formation reconfiguration is introduced, including the dynamic model and the nonlinear optimal control problem. In section 3, the programming and design of the fast spacecraft formation reconfiguration problem is taken as a multi-layer optimization problem. An adaptive surrogate model is proposed for solving the multi-layer optimization problem in section 4. In section 5, the accuracy, efficiency and robustness of the proposed adaptive surrogate model are verified through the numerical simulations of spacecraft formation reconfiguration.

\section{Background}

In this section, we shall give a brief introduction to some basic knowledge used for constructing fast path planning models for spacecraft formation reconfiguration on libration point orbits. First, the dynamic model for the spacecraft formation will be introduced. Then, the path planning problem of spacecraft formation reconfiguration will be formulated as a nonlinear optimal control problem.

\subsection{Dynamic model}

The primary concern in this paper is the spacecraft formation reconfiguration problem on libration point orbits in the sun-earth system. Therefore, the circular restricted three-body problem (CRTBP) model is used to construct the dynamical equations for the spacecraft formation reconfiguration problem. In the CRTBP model, there are two massive bodies (i.e., the sun and the earth) and a spacecraft. The sun and the earth are assumed to be in circular orbit around the barycenter of the 
sun-earth system because their masses are far larger than that of the spacecraft and the motion of the two primary bodies is not affected by the spacecraft. Because we mainly discuss the orbits around the second libration $\left(L_{2}\right)$ point, we set the origin of the reference coordinate system at the $L_{2}$ point. The $\mathrm{x}$ vector of the reference coordinate is extended from the sun to the earth, the y vector is normal to the $\mathrm{x}$ vector and in the rotation plane, and the $\mathrm{z}$ vector complies with the rule of right-handed frames. Furthermore, continuous low thrust (typically electrically powered spacecraft propulsion) has been developed and employed as a novel propulsion system for deep space exploration in recent years. The present study also employs continuous low thrust for spacecraft formation reconfiguration.

Therefore, according to the above assumptions and statements, we can obtain the controlled dynamic equations for the $i$ th spacecraft in the following dimensionless form [1]:

$$
\begin{gathered}
\ddot{x}^{i}-2 \dot{y}^{i}-x^{i}=-\frac{(1-\mu)\left(x^{i}+1+1 / \gamma\right)}{\gamma^{3} d_{1}^{3}}-\frac{\mu\left(x^{i}+1\right)}{\gamma^{3} d_{2}^{3}}+\frac{1-\mu+\gamma}{\gamma}+u_{x}^{i} \\
\ddot{y}^{i}+2 \dot{x}^{i}-y^{i}=-\frac{(1-\mu) y^{i}}{\gamma^{3} d_{1}^{3}}-\frac{\mu y^{i}}{\gamma^{3} d_{2}^{3}}+u_{y}^{i} \\
\ddot{z}^{i}=-\frac{(1-\mu) y^{i}}{\gamma^{3} d_{1}^{3}}-\frac{\mu z^{i}}{\gamma^{3} d_{2}^{3}}+u_{z}^{i}
\end{gathered}
$$

where the dot represents the time derivative in the rotating frame, $i=1,2, \cdots, n, n$ is the number of spacecraft in the geometric formation, $\mu$ is the ratio of the earth's mass to the sum of the masses of both the earth and the sun, $d_{1}=\sqrt{\left(x^{i}+1+1 / \gamma\right)^{2}+\left(y^{i}\right)^{2}+\left(z^{i}\right)^{2}}$, and $d_{2}=\sqrt{\left(x^{i}+1\right)^{2}+\left(y^{i}\right)^{2}+\left(z^{i}\right)^{2}}$. The distance between the earth and the $L_{2}$ libration point is denoted by $\gamma$. The symbols $u_{x}^{i}, u_{y}^{i}$ and $u_{z}^{i}$ are the control inputs of the $i$ th controlled spacecraft in the $x, y$, and $z$ directions.

It is well known that the collinear libration points have families of periodic and quasi-periodic unstable orbits. Halo orbits are special periodic orbits around the collinear libration points. The path planning problem for spacecraft formation reconfiguration in this paper is studied on halo orbits. Because no analytical solution exists for halo orbits, the numerical solution of the halo orbits should be computed before the implementation of the spacecraft formation reconfiguration. To obtain the numerical solution of a halo orbit, a third-order approximate analytic solution [17] based differential correction algorithm [18] is generally employed to compute the initial conditions of the halo orbits. 


\subsection{Nonlinear optimal control problem}

Although several path planning methods have been developed, such as the artificial potential field method and the sample-based planning method, optimal control theory is the most natural framework for solving the path planning problem of spacecraft formation reconfiguration [19]. Therefore, to obtain accurate quantitative results, the present path planning problem is formulated as a nonlinear optimal control problem. Fig. 1 shows a diagram of spacecraft formation reconfiguration near the libration point orbit.
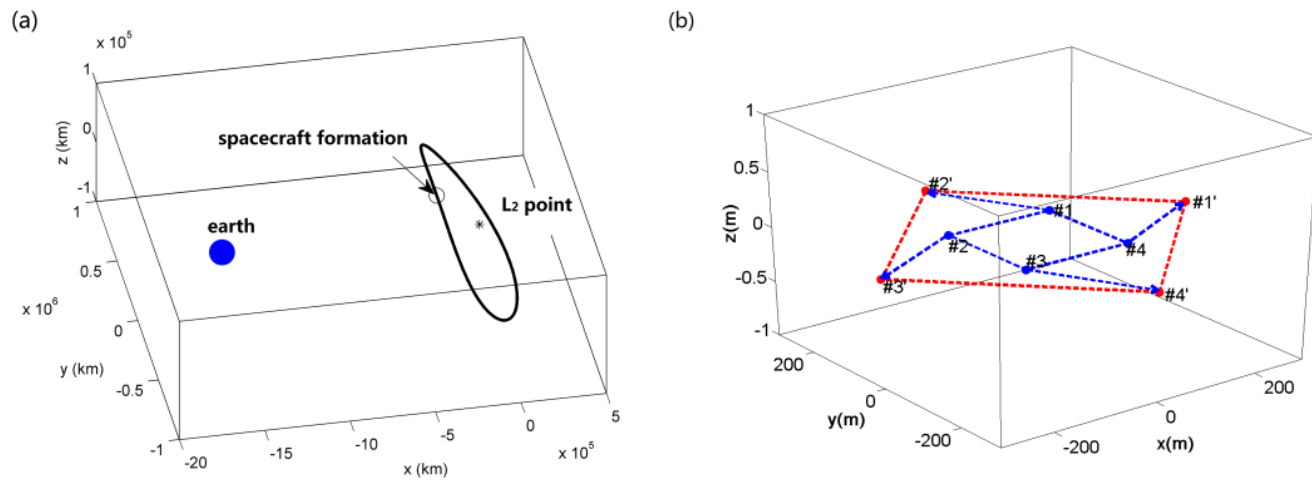

Fig.1.Spacecraft formation reconfiguration near the libration point orbit: (a) global view; (b) local view

Therefore, the path planning problem for spacecraft formation reconfiguration in terms of nonlinear optimal control can be stated as follows: the spacecraft with the controlled nonlinear differential equation in the form of state space Eq. (4) is derived by continuous low-thrust $\mathbf{u}$ from the initial formation $\mathbf{x}_{0}$ to the final formation $\mathbf{x}_{f}$ :

$$
\left\{\begin{array}{l}
\dot{\mathbf{x}}^{(i)}=\mathbf{f}\left(\mathbf{x}^{(i)}(\mathrm{t}), \mathbf{u}^{(i)}(\mathrm{t}), \mathrm{t}\right) \\
\mathbf{x}^{(i)}\left(\mathrm{t}_{0}\right)=\mathbf{x}_{0}^{(i)} ; \mathbf{x}^{(i)}\left(\mathrm{t}_{f}\right)=\mathbf{x}_{f}^{(i)}
\end{array}\right.
$$

where the state vector is $\mathbf{x}^{(i)}=\left(x^{i}, y^{i}, z^{i}, \dot{x}^{i}, \dot{y}^{i}, \dot{z}^{i}\right)^{\mathrm{T}}$ and the control vector is $\mathbf{u}^{(i)}=\left(u_{x}^{i}, u_{y}^{i}, u_{z}^{i}\right)^{\mathrm{T}}$.

Meanwhile, the following performance index $J$ under the constraints of nonlinear differential Eq. (4) should be minimized:

$$
J=\frac{1}{2}\left[\mathbf{M}_{\mathrm{d}} \mathbf{x}\left(t_{f}\right)-\Psi\right]^{\mathrm{T}} \mathbf{S}_{\mathrm{d}}\left[\mathbf{M}_{\mathrm{d}} \mathbf{x}\left(t_{f}\right)-\Psi\right]+\frac{1}{2} \int_{t_{o}}^{t_{f}}\left[\left(\mathbf{x}-\mathbf{x}_{d}\right)^{\mathrm{T}} \mathbf{Q}\left(\mathbf{x}-\mathbf{x}_{d}\right)+\left(\mathbf{u}-\mathbf{u}_{d}\right)^{\mathrm{T}} \mathbf{R}\left(\mathbf{u}-\mathbf{u}_{d}\right)\right] \mathrm{dt}
$$

where $\mathbf{x}_{d}=\mathbf{x}_{f}$ and $\mathbf{u}_{d}$ are the desired formation and control inputs during the process of reconfiguration, respectively. $\mathbf{Q}$ and $\mathbf{S}_{\mathrm{d}}$ are positive semidefinite matrices, and $\mathbf{R}$ is a positive definite 
matrix. $\quad \mathbf{M}_{\mathrm{d}}=\mathbf{I}_{d}$ and $\Psi=\mathbf{x}_{f}$ are the given identity matrix and desired vector. $t_{0}$ and $t_{f}$ are the initial and final times. The terminal cost shows that the spacecraft should be arrived at desired state at final time $t_{f}$. The essence of integration part is a regulator, i.e., from the initial departures to the desired zero departures. In this paper, the above nonlinear optimal control problem with the given known parameters will be solved by the recently proposed symplectic algorithms [20][21].

From the above statement of the nonlinear optimal control problem, we can see that quantitative numerical results can only be obtained by solving the above nonlinear optimal control problem with the given parameters, for example, the beginning time $t_{0}$ for reconfiguration, the ending time $t_{f}$ for reconfiguration, the initial formation state $\mathbf{x}_{0}$ and the desired formation state $\mathbf{x}_{f}$. The above parameters can be mainly determined based on realistic conditions and the practical mission. Because the above parameters can be selected from a wide variety, the most important problem is how to select the optimal parameters effectively for path planning of spacecraft formation reconfiguration under extreme conditions.

\section{Programming and design of fast reconfiguration problem}

To obtain the objective of maximizing the area of the spacecraft formation under a geometric constraint for some abnormal conditions and/or emergency cases, in this section, we formalize the fast spacecraft formation reconfiguration problem as a multi-layer optimization model. Thus far, it makes the most sense to find the maximized area of the spacecraft formation under the geometric constraint by using the parameter optimization method. With the results of parameter optimization, we can obtain the desired new formation. Meanwhile, with the given states of each spacecraft in the new desired formation, the nonlinear optimal control method can be employed for solving the path planning problem. Essentially, this multi-layer optimization problem contains two loops, i.e., an outer loop and an inner loop. In the outer loop, the nonlinear optimal control problem is taken as a dynamical optimization to be solved; i.e., with the given states of each spacecraft, fuel consumption should be minimized in the process of spacecraft formation reconfiguration. In the inner loop, the parameter optimization problem, taken as a static optimization, should be solved; i.e., within the geometric constraint, the maximized area of the spacecraft formation is the primary objective. 


\subsection{Multi-layer optimization model}

There are two performance indexes in the present spacecraft formation reconfiguration problem. One is velocity change ( $\Delta v)$, which is taken as an approximate performance for evaluating fuel or cost consumption [22], and it can be obtained from the numerical results of nonlinear optimal control problem. It is noted that the real fuel consumption is related to both the mass of spacecraft and its velocity increment $(\Delta v)$. In general, a heavier spacecraft spends more fuel than a lighter one for the same $\Delta v$. In this paper, the mass of all spacecraft is given to be equal, and so it is reasonable to use the sum of velocity change of the spacecraft formation instead of real fuel consumption. The other one is the maximized area $s$ surrounded by the spacecraft formation. These two performance indexes are not parallel in optimization, and they have a multi-layer optimization structure. Considering the spacecraft formation reconfiguration in the $x-y$ plane in this paper, the multi-layer optimization model can be described as follows:

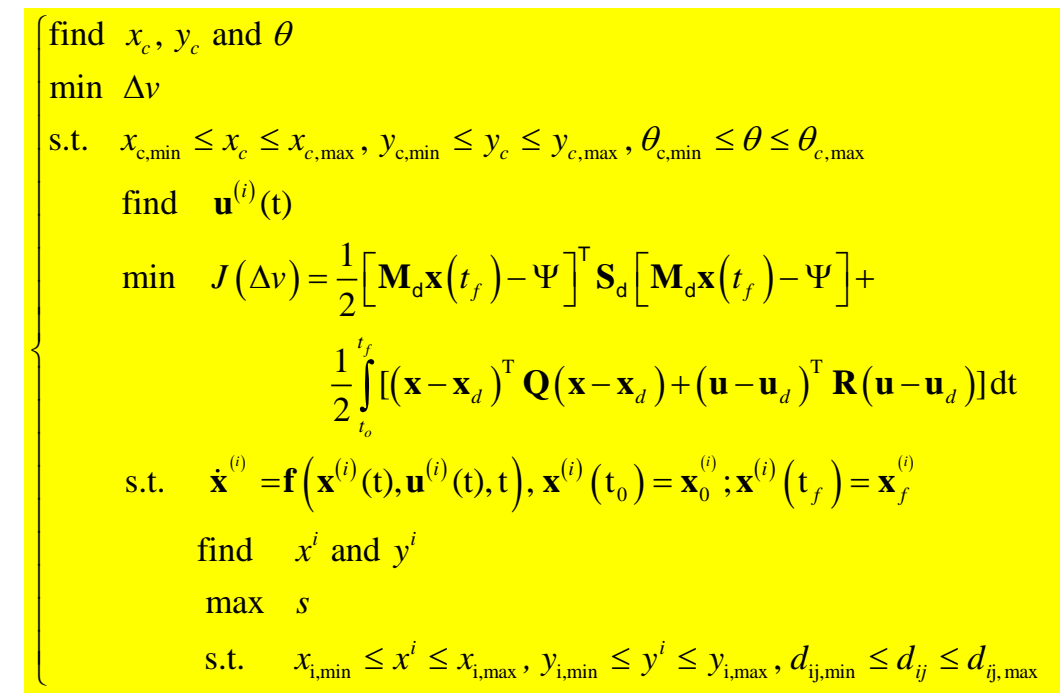

where $x_{c}, y_{c}$ and $\theta$ are the center coordinates and rotation angle of the plane spacecraft formation in local coordinates. $x_{c, \text { min }}, x_{c, \max }, y_{c, \min }, y_{c, \max }, \theta_{c, \min }$ and $\theta_{c, \max }$ are the minimum and maximum values of the center coordinates $x_{c}, y_{c}$ and the rotation angle $\theta . x_{i}$ and $y_{i}$ are the positions coordinates of the $i$ th spacecraft in the formation. $x_{\mathrm{i}, \min }, x_{i, \max }, y_{\mathrm{i}, \min }$ and $y_{i, \max }$ are the minimum and maximum values of the $i$ th spacecraft in local coordinates. $d_{i j}=\sqrt{\left(x^{i}-x^{j}\right)^{2}+\left(y^{i}-y^{j}\right)^{2}},(i \neq j)$ is the distance between the $i$ th spacecraft and $j$ th spacecraft. $d_{\mathrm{ij}, \min }$ and $d_{i j, \max }$ are the minimum and maximum distances between the $i$ th spacecraft and $j$ th spacecraft. 
From Eq.(6), the multi-layer optimization model is characterized by the existence of two optimization problems. The purpose of proposing the above multi-layer optimization model is to address decision processes involving two decision-makers within a hierarchical structure. The second level optimizes its objective function under the given parameters from the first level. In turn, the first level, with complete information on the possible reactions of the second level, selects parameters to optimize its own objective function [23]. From the spacecraft formation reconfiguration point of view, Eq. (6) means that the fuel consumption $\Delta v$ of the spacecraft formation reconfiguration is minimized under the maximized area $s$ surrounded by the spacecraft formation. With the solution of the inner-loop optimization problem, i.e., the position coordinates of each spacecraft for maximizing the formation area, the minimized fuel consumption can be obtained by the outer-loop optimization. In particular, the nonlinear optimal control problem Eqs.(4)(5) is solved for thousands of possible position coordinate of the spacecraft formation in the process of optimization. Particularly for exhustive search algorithm, the computational cost and time-consuming of exhustive search algorithm are unacceptable. To implement the above multi-layer optimization, detailed explanations regarding the inner-loop and outer-loop optimizations are given in the following two parts

\subsection{Inner-loop optimization}

The geometrical area surrounded by the spacecraft is considered as an inner-loop optimization problem, and it can be rewritten as follows:

$$
\begin{cases}\text { find } & x^{i} \text { and } y^{i} \\ \max & s \\ \text { s.t. } & x_{\mathrm{i}, \min } \leq x^{i} \leq x_{\mathrm{i}, \max } \\ & y_{\mathrm{i}, \min } \leq y^{i} \leq y_{\mathrm{i}, \max } \\ & d_{\mathrm{i} \mathrm{j}, \min } \leq d_{i j} \leq d_{i \mathrm{j}, \max }\end{cases}
$$

Due to the number of spacecraft in the geometrical formation, i.e., $i=1,2, \cdots, n$, there are $2 n$ design variables for the above inner-loop optimization. The geometry of the spacecraft formation in the $x-y$ plane is first divided into several triangles, and then, the area of every triangle can be computed using the coordinates of the corner spacecraft. Finally, the whole geometrical area of the spacecraft formation can be obtained by summing all the triangles. In this paper, we employ Matlab function "DelaunayTri" to create a delaunay triangulation from a set of coordinates of the corner spacecraft. Once the geometrical shape of the spacecraft formation is obtained by solving this inner-loop 
optimization problem, the number of design variables from coordinates $x^{i}$ and $y^{i}$ can be reduced to $x_{c}, y_{c}$ and $\theta$. Table 1 shows the maximum area for a number of different spacecraft formations from $d_{\mathrm{i}, \text { min }}=20$ meters to $d_{\mathrm{ij}, \text { max }}=1000$ meters. When the number of spacecraft in the formation is odd, the optimal solution is an equilateral polygon. Otherwise, the optimal solution for the spacecraft formation is a polygon with a symmetry axis.

Table 1 The max area of different spacecraft formations in the range of $20 \mathrm{~m} \leq d_{i j} \leq 1000 \mathrm{~m}$.

\begin{tabular}{lllllll}
\hline Number of spacecraft & 5 & 6 & 7 & 8 & 9 & 10 \\
\hline Max area of formation $\left(10^{5} \mathrm{~m}^{2}\right)$ & 6.5716 & 6.7498 & 7.1974 & 7.2687 & 7.4562 & 7.4914 \\
\hline
\end{tabular}

\subsection{Outer-loop optimization}

The minimized fuel consumption of the spacecraft formation reconfiguration is considered as an outer-loop optimization problem. In the outer-loop optimization, the main mission is to determine the fuel consumption of the spacecraft formation reconfiguration under the condition of maximized formation geometrical area. However, the relationship between the design variables and the objective function is an implicit nonlinear mapping. With one group of given design variables $x_{c}, y_{c}$ and $\theta$, the fuel consumption $\Delta v$ should be computed as a nonlinear optimal control problem. Furthermore, the essence of the nonlinear optimal control problem is a dynamic optimization. Therefore, the outer-loop optimization can be expanded into the following formula.

$$
\left\{\begin{array}{l}
\text { find } x_{c}, y_{c} \text { and } \theta \\
\min \Delta_{c} \\
\text { s.t. } \quad x_{c, \text { min }} \leq x_{c} \leq x_{c, \text { max }} \\
y_{c, \text { min }} \leq y_{c} \leq y_{c, \text { max }} \\
\theta_{c, \text { min }} \leq \theta \leq \theta_{c, \text { max }} \\
\text { find } \quad \mathbf{u}^{(i)}(\mathrm{t}) \\
\min \quad J(\Delta v)=\frac{1}{2}\left[\mathbf{M}_{\mathrm{d}} \mathbf{x}\left(t_{f}\right)-\Psi\right]^{\mathrm{T}} \mathbf{S}_{\mathrm{d}}\left[\mathbf{M}_{\mathrm{d}} \mathbf{x}\left(t_{f}\right)-\Psi\right]+ \\
\quad \frac{1}{2} \int_{t_{o}}^{t_{f}}\left[\left(\mathbf{x}-\mathbf{x}_{d}\right)^{\mathrm{T}} \mathbf{Q}\left(\mathbf{x}-\mathbf{x}_{d}\right)+\left(\mathbf{u}-\mathbf{u}_{d}\right)^{\mathrm{T}} \mathbf{R}\left(\mathbf{u}-\mathbf{u}_{d}\right)\right] \mathrm{dt} \\
\text { s.t. } \quad \dot{\mathbf{x}}^{(i)}=\mathbf{f}\left(\mathbf{x}^{(i)}(\mathrm{t}), \mathbf{u}^{(i)}(\mathrm{t}), \mathrm{t}\right), \mathbf{x}^{(i)}\left(\mathrm{t}_{0}\right)=\mathbf{x}_{0}^{(i)} ; \mathbf{x}^{(i)}\left(\mathrm{t}_{f}\right)=\mathbf{x}_{f}^{(i)}
\end{array}\right.
$$

Eq. (8) is also a multi-layer optimization problem. The nonlinear optimal control problem should be solved under the given parameters $x_{c}, y_{c}$ and $\theta$. Different parameters $x_{c}, y_{c}$ and $\theta$ can produce 
different fuel consumption $\Delta v$. Though the geometrical shape and position of the spacecraft formation after reconfiguration can be determined from the above optimization, the corresponding relationship between the initial formation of the spacecraft and the final formation is still a problem. Every spacecraft in the initial formation can be transferred into every corner position of the final formation. Therefore, the corresponding relationship is a combinatorial optimization problem. For each pair of initial and final positions of the spacecraft, one nonlinear optimal control problem should be solved. In fact, too much computational cost would be needed to evaluate every combinatorial solution. Therefore, we should make a reasonable corresponding relationship for removing obviously unreasonable combinations to increase the computational efficiency.

The main idea of this reasonable corresponding relationship can be explained as follows. First, the numbers of all spacecraft are arranged in counterclockwise order. Then, we assume that there are no intersections between any two paths. Fig. 2 shows the quick look interpretation of the spacecraft formation reconfiguration for the intersection case and the no intersection case. Because most paths of the spacecraft formation reconfigurations have the shortest path in the optimum solution, the intersection cases will increase the length of the path and the fuel consumption [24][25]. Finally, based on the above reasonable corresponding relationship, the optimal solution of each spacecraft's transferring path can be obtained by solving one nonlinear optimal control problem.

(a)

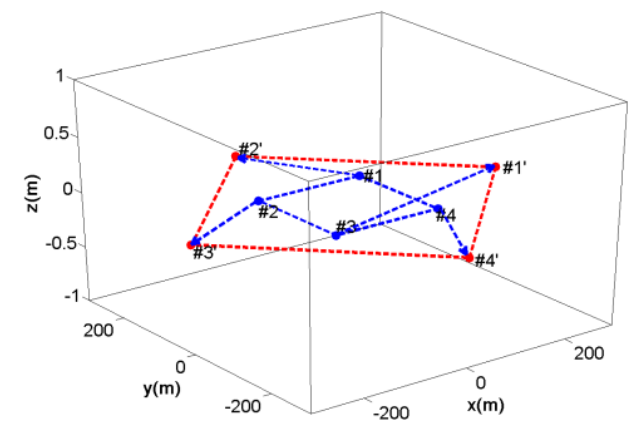

(b)

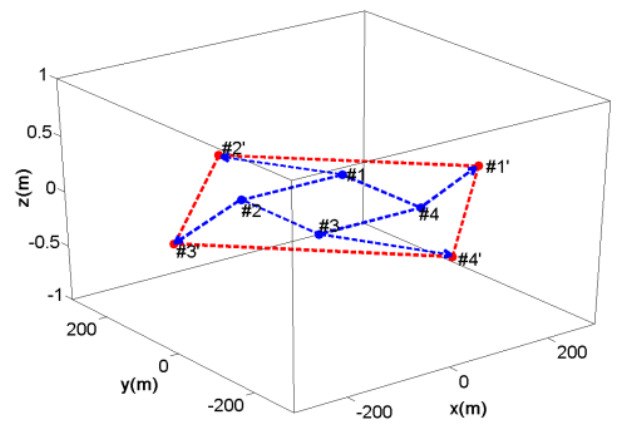

Fig. 2. Spacecraft formation reconfiguration: (a) intersection case; (b) no intersection case

As a final consideration, even after the reasonable corresponding relationship has been introduced into the above multi-layer optimization model, the computational cost of the multi-layer optimization model, which contains enormous numbers of nonlinear optimal control problems, is still an important problem. If the direct search method or direct optimization method is employed for solving the above multi-layer optimization problem, the computational time is unacceptable for fast spacecraft formation reconfiguration. Therefore, an efficient optimization strategy must be designed to solve the 
above multi-layer optimization problem for fast spacecraft formation reconfiguration missions.

\section{Adaptive surrogate model}

With regard to the above multi-layer optimization model with numerous nonlinear optimal control problems, the enormous number of nonlinear optimal control problems has great influence on the efficiency of the optimization process. Furthermore, more emphasis needs to be placed on the efficiency of solving the above multi-layer optimization model, that is, to rapidly obtain a new and effective formation in abnormal conditions and/or emergency cases. Therefore, to reduce the computational cost, the framework of an adaptive surrogate model optimization that decomposes the original optimization into two steps is proposed in this section. Through the proposed adaptive surrogate model optimization, the optimum results for fast spacecraft formation reconfiguration can be achieved with less computational cost.

\subsection{Uniform design of experiments}

The surrogate model is a mathematical model that approximates the multivariate input/output behavior of complex systems, constructed by the input/output relationships of sample points derived from the design of experiments (DOE) [26], such as orthogonal array design, central composite design, Latin hypercube sampling and optimal LHS [16]. In this paper, the uniform DOE [27] is used to obtain the initial samples and updated samples. The uniform DOE with 30 sample points is selected to obtain the initial design, and then, the uniform DOE with 15 new sample points is selected to obtain the updated design. More information regarding the uniform DOE can be found in Ref. [27].

\subsection{Construction of the surrogate model}

There are many methods for constructing the surrogate model, for example, the polynomial regression method, the Kriging method and the radial basis functions (RBF) method. In this paper, the Kriging and radial basis functions methods are employed to construct the surrogate model.

\subsubsection{The Kriging method}

The Kriging model is one of the most popular surrogate models, and it can simulate the true function response by using a series of known samples. These sample points which are generated by 
design of experiment contains known design variables and their objective values. The true function can be written as follows [28][29]:

$$
\mathbf{y}(\mathbf{s})=\sum_{i=1}^{k} \beta_{i} \mathbf{f}_{i}(\mathbf{s})+\varepsilon(\mathbf{s})
$$

In Eq. (9), the first term $\sum_{i=1}^{k} \beta_{i} \mathbf{f}_{i}(\mathbf{s})$ represents the mean value, and it can be expressed by the linear combination of the known functions. $\beta_{i}$ is the unknown coefficient, $\mathbf{f}_{i}(\mathbf{s})$ is the known function, and $k$ is the number of sample points. The second term $\varepsilon(\mathbf{s})$ is the error term for the approximate function $\hat{\mathbf{y}}(\mathbf{s})$. The random error $\varepsilon(\mathbf{s})$ has the following two properties [30]:

$$
\begin{gathered}
E[\varepsilon(\mathbf{s})]=0 \\
\operatorname{Cov}\left[\varepsilon\left(\mathbf{s}^{(i)}\right), \varepsilon\left(\mathbf{s}^{(j)}\right)\right]=\sigma^{2} \mathbf{R}\left[R\left(\mathbf{s}^{(i)}, \mathbf{s}^{(j)}\right)\right]
\end{gathered}
$$

From Eqs.(10) and (11), the random error $\varepsilon(\mathbf{s})$ is a Gaussian random function with zero mean and non-zero variance. $\mathbf{R}$ is the correction matrix between the sample points, and $R\left(\mathbf{s}^{(i)}, \mathbf{s}^{(j)}\right)$ is a basis function. The form of the basis function can be Gaussian, exponential, linear and so on. The accuracy of Kriging model has a little difference according to different basis functions. Meanwhile, different problems with the same basis function also have different numerical accuracies. Therefore, we have done some numerical tests for comparison accuracy of different basis functions, and we find that an exponential function with parameter $\theta_{n}$ is more suitable for the present problem and chosen in this paper; its expression can be written as:

$$
R\left(\mathbf{s}^{(i)}, \mathbf{s}^{(j)}\right)=\exp \left[-\sum_{n=1}^{k} \theta_{n}\left|\mathbf{s}_{n}^{(i)}-\mathbf{s}_{n}^{(j)}\right|^{2}\right]
$$

By a series of formulas, the Kriging approximation is typically formulated as follows:

$$
\hat{\mathbf{y}}(\mathbf{s})=\mathbf{f}^{\mathrm{T}} \boldsymbol{\beta}+\boldsymbol{r}^{\mathrm{T}} \mathbf{R}^{-1}(\mathbf{y}-\mathbf{f} \boldsymbol{\beta})
$$

where $\boldsymbol{\beta}$ is the coefficient matrix, $\mathbf{r}$ is the correlation vector between any new point and the original sample points, $\mathbf{f}$ is the regression function, and $\hat{\mathbf{y}}(\mathbf{s})$ is the predicted function.

Once the basis function and the regression function are known, the Kriging model can be determined. 


\subsubsection{The radial basis function method}

The radial basis function (RBF) method uses a linear combination of the basis functions to express the objective function as follows [23]:

$$
\hat{\mathbf{y}}(\mathbf{x})=\sum_{i=1}^{k} \beta_{i} \phi_{i}\left(\left\|\mathbf{S}-\mathbf{s}_{i}\right\|\right)=\boldsymbol{\beta}^{\mathrm{T}} \boldsymbol{\phi}
$$

$k$ is equal to the number of sample points, $\beta_{i}$ is the coefficient of the basis function $\phi_{i}$, and $\left\|\mathbf{s}-\mathbf{s}_{i}\right\|$ represents the Euclidean distance from the predicted point $\mathbf{s}$ to the sample point $\mathbf{s}_{i}$. The basis function $\phi_{i}$ can be chosen as a Gaussian function, an exponential function, a multi-quadric function, etc. The exponential function is chosen in this paper.

With the RBF given in Eq. (14), the interpolation condition is satisfied as follows:

$$
\hat{\mathbf{y}}\left(\mathbf{s}_{i}\right)=\mathbf{y}\left(\mathbf{s}_{i}\right)
$$

where $\hat{\mathbf{y}}\left(\mathbf{s}_{i}\right)$ is the predicted value at the evaluated sample point $\mathbf{s}_{i}$ and $\mathbf{y}\left(\mathbf{s}_{i}\right)$ is the exact solution at the evaluated sample point $\mathbf{s}_{i}$.

With the interpolation condition Eq. (15), the RBF in Eq. (14) can be rewritten as follows:

$$
\begin{gathered}
\mathbf{A} \boldsymbol{\beta}=\mathbf{y} \\
\mathbf{A}=\left[\begin{array}{ccc}
\phi\left(\left\|\mathbf{s}_{1}-\mathbf{s}_{l}\right\|\right) & \ldots & \phi\left(\left\|\mathbf{s}_{1}-\mathbf{s}_{k}\right\|\right) \\
\vdots & \ddots & \vdots \\
\phi\left(\left\|\mathbf{s}_{k}-\mathbf{s}_{l}\right\|\right) & \ldots & \phi\left(\left\|\mathbf{s}_{k}-\mathbf{s}_{k}\right\|\right)
\end{array}\right] \\
\mathbf{y}=\left[\begin{array}{c}
\mathbf{y}\left(\mathbf{s}_{l}\right) \\
\vdots \\
\mathbf{y}\left(\mathbf{s}_{k}\right)
\end{array}\right]
\end{gathered}
$$

From the new expression for RBF, Eqs. (16) (18), the unknown coefficient matrix $\beta$ can be determined by solving the above linear equation:

$$
\boldsymbol{\beta}=\mathbf{A}^{-1} \mathbf{y}
$$

Though RBF is just a linear combination of basis functions, the RBF can also predict the nonlinear response of the optimization system by the choice of nonlinear basis functions.[16]

\subsection{A brief review of ant colony optimization}

Once a surrogate model is successfully constructed using the DOE, various specialized 
optimization algorithms can be employed to perform the SBO. Because this paper employs the ant colony optimization (ACO) method for adaptive surrogate model optimization, a brief review of ACO is given here for a better understanding. ACO is a stochastic global search method that mimics some natural aspects of ants finding food. ACO operates on ants by applying the principle of memory operating characteristics to converge to an optimal solution. The ants leave pheromones along their path, and additional pheromones are left when the ants find food. After one ant finds food, more ants will come to obtain the pheromones. In the end, most ants will find food through the best path. This method is chosen in this paper for its great ability to obtain the global optimal result, and we employed ACO to obtain the global optimal result of the adaptive surrogate model. More information on ACO may be found in reference [12], among others.

\subsection{Convergence criterion}

Actually, neither Kriging nor RBF can give a satisfactory surrogate model for all the spacecraft formation reconfiguration missions; for example, the main drawbacks of the Kriging method are as follows: if any of the sample points used to construct the model are too close, the correlation matrix can become singular, and large amounts of computer memory and CPU time are needed for constructing models with high dimensionality or large sample sets [23]. Therefore, we propose an adaptive surrogate model in this paper. To successfully implement the adaptive process, the convergence criterion should be given first.

The relative error (RE), the absolute error (AE) and the generalized absolute error (GAE), respectively, can be defined as follows.

$$
\begin{gathered}
R E=\left|\left(\mathbf{s}^{i+1}-\mathbf{s}^{i}\right) / \mathbf{s}^{i+1}\right| \times 100 \% \\
A E=\left|\mathbf{s}^{i+1}-\mathbf{s}^{i}\right| \\
G A E=\left|\left(\mathbf{s}^{i+1}-\mathbf{s}^{i}\right) / R\right| \times 100 \%
\end{gathered}
$$

where $\mathbf{s}^{i}$ is the optimal design variable in the $i$ th iteration, $\mathbf{x}^{i+1}$ is the optimal design variable in the $i+1$ th iteration, and $R$ is the changing range of the design variable $\mathbf{x}$.

The maximum relative error (MRE) and the maximum generalized absolute error (MGAE), respectively, are defined as follows: 


$$
\begin{aligned}
& M R E=\max \left(\left|\left(\mathrm{s}_{1}^{i+1}-\mathrm{s}_{1}^{i}\right) / \mathrm{s}_{1}^{i+1},\left(\mathrm{~s}_{2}^{i+1}-\mathrm{s}_{2}^{i}\right) / \mathrm{s}_{2}^{i+1},\left(\mathrm{~s}_{n}^{i+1}-\mathrm{s}_{n}^{i}\right) / \mathrm{s}_{n}^{i+1},\left(y^{i+1}-y^{i}\right) / y^{i+1}\right|\right) \times 100 \% \\
& M G A E=\max \left(\left|\left(\mathrm{s}_{1}^{i+1}-\mathrm{s}_{1}^{i}\right) / R_{1},\left(\mathrm{~s}_{2}^{i+1}-\mathrm{s}_{2}^{i}\right) / R_{2},\left(\mathrm{~s}_{n}^{i+1}-\mathrm{s}_{n}^{i}\right) / R_{n},\left(y^{i+1}-y^{i}\right) / R_{n+1}\right|\right) \times 100 \%
\end{aligned}
$$

If the convergence accuracy $\varepsilon$ and $\mathrm{E}$ (small constant values) are given in the iteration process, then the adaptive process can be terminated for $\mathrm{MRE} \leq \varepsilon$ or $\mathrm{MGAE} \leq \mathrm{E}$.

\subsection{Implementation of the adaptive surrogate model}

The previous subsections have given descriptions of the DOE, the Kriging and RBF methods, the ACO method and the convergence criterion. In this subsection, we will propose the detailed implementation process for the adaptive surrogate model using these basic tools.

Fig. 3 shows the flow chart of the proposed adaptive surrogate model. The framework of the present adaptive surrogate model optimization contains two sublevel optimizations. In the first step, 30 initial samples are generated by using the uniform design of experiments, and then, the surrogate model is constructed by employing the Kriging and RBF methods. The ACO method is then used to obtain the global optimum solution of the current surrogate model. If the MRE is less than $\varepsilon$ or the MGAE is less than E, satisfactory convergence accuracy has been obtained. However, if the convergence accuracy is not satisfied at the present iteration step, 15 new samples from the uniform DOE are added around the current global optimum point, and then, the surrogate model is updated in the first step. The above process is constantly repeated until it reaches the convergence criterion. In the iteration process, parameter $\varepsilon$ is set to 1 and parameter $\mathrm{E}$ is set to 0.2 . In the second step, with the convergence result at the first step as a preliminary result, the range of the design variables is reduced to $10 \%$ of the original range. Thirty new samples are generated within the reduced range to obtain the sample points of the second step. If the convergence criterion cannot be satisfied by the initial sample points, another 15 new sample points of the uniform DOE are updated until the convergence criterion is satisfied. In the second step, parameter $\varepsilon$ is set to be 0.5 and parameter $\mathrm{E}$ is set to be 0.1 . Through the above two steps, the adaptive surrogate model can obtain more accurate results. 


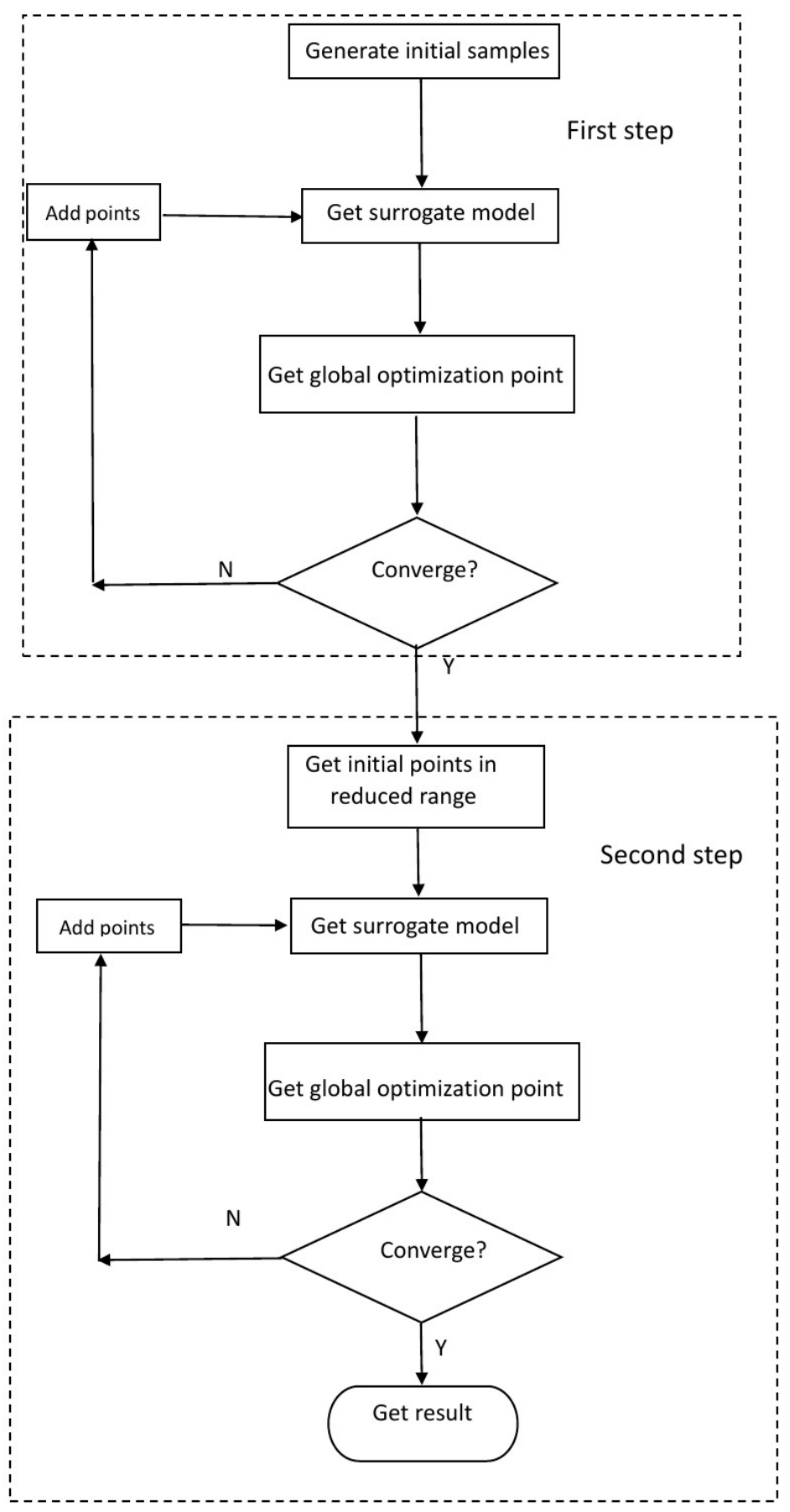

Fig. 3. Flow chart for generating the adaptive surrogate model

\section{Results and discussions}

In this section, fast spacecraft formation reconfiguration based on an adaptive surrogate model for a given three-dimensional periodic halo orbit in the sun-earth system around the $L_{2}$ point is implemented by numerical simulations. In particular, formations with five and six spacecraft are taken as examples to show the accuracy, efficiency and parameter influences of the proposed adaptive 
surrogate model in the extreme cases of reconfigurations. Moreover, to show the accuracy and efficiency of the proposed adaptive surrogate model, the ACO based on the direct parameter optimization model is also employed to solve the same spacecraft formation reconfiguration problem. All numerical simulations in this section were performed using MATLAB software (R2009a on an Intel Core i5 2.5 GHz CPU with 2G RAM running under Windows 7).

The initial halo orbit parameters for spacecraft formation reconfiguration are given in Table 2 . When the spacecraft formation is overtaken by bad conditions, the specific instantaneous positions of the spacecraft formation reconfiguration in local coordinates are as given in Table 3 . The present starting time for spacecraft formation reconfiguration in the numerical simulation is set to zero at the initial position. The simulation time is divided into a series of equal intervals, and the length of step $\eta=\left(t_{f}-t_{0}\right) / N_{c} \quad\left(N_{c}=1000\right.$ in this paper $)$. The mass of each spacecraft is $1000 \mathrm{~kg}$. Thus far, all parameters for numerical simulations have been given in the previous tables.

Table 2 Initial conditions of the given halo orbit for spacecraft formation reconfiguration

\begin{tabular}{lll}
\hline Parameter & Value & Unit \\
\hline$x_{0}$ & $-2.78175218809931 \times 10^{8}$ & $\mathrm{~m}$ \\
$y_{0}$ & 0 & $\mathrm{~m}$ \\
$z_{0}$ & $2.00514873577280 \times 10^{8}$ & $\mathrm{~m}$ \\
$\dot{x}_{0}$ & 0 & $\mathrm{~m} / \mathrm{s}$ \\
$\dot{y}_{0}$ & $3.115796960140503 \times 10^{2}$ & $\mathrm{~m} / \mathrm{s}$ \\
$\dot{z}_{0}$ & 0 & $\mathrm{~m} / \mathrm{s}$ \\
\hline
\end{tabular}

Table 3 The specific initial positions of the spacecraft formation reconfiguration in local coordinates

\begin{tabular}{llllllll}
\hline Reconfiguration cases & Position & $\# 1$ & $\# 2$ & $\# 3$ & $\# 4$ & $\# 5$ & $\# 6$ \\
\hline \multirow{2}{*}{5 spacecraft } & $\mathrm{X}(\mathrm{m})$ & 500.000 & 250.000 & -250.000 & -250.000 & 250.000 & --- \\
& $\mathrm{Y}(\mathrm{m})$ & 0.000 & 433.013 & 433.013 & -433.013 & -433.013 & --- \\
& $\mathrm{X}(\mathrm{m})$ & 415.304 & 102.571 & -287.400 & -287.400 & 102.571 & 415.211 \\
6 spacecraft & $\mathrm{Y}(\mathrm{m})$ & 200.000 & 449.396 & 360.388 & -360.388 & -449.396 & -200.000 \\
& & & & & & & \\
\end{tabular}




\section{1.}

\section{Accuracy}

In this subsection, we will focus our attention on the accuracy of the proposed adaptive surrogate model. With the above known information, the fast spacecraft formation reconfiguration based on the adaptive surrogate model has been successfully implemented by numerical simulations. Meanwhile, the reconfiguration time is set to be 1 hour for all the different formation reconfiguration cases. Moreover, to eliminate the symmetry of the final spacecraft formation, the range of local coordinate $\theta$ is changed from $0-2 \pi$ to $0-\pi$. In the proposed adaptive surrogate model, the barycentric coordinates of the final formation $\left(x_{c}\right.$ and $\left.y_{c}\right)$ and the rotation of the final formation $(\theta)$ are taken as the input variables. The velocity increment $(\Delta v)$, which represents the fuel consumption, is taken as the output variable. To determine optimal reconfiguration trajectories for all spacecraft, the coordinates of each spacecraft after reconfiguration have been calculated from the barycentric coordinates and rotation angle for the final formation. As a result, Table 4 shows the specific final positions obtained from the adaptive surrogate model optimization for the optimal spacecraft reconfiguration in local coordinates. Fig. 4 shows the optimal trajectory of every spacecraft during reconfiguration.

As seen from Fig. 4, the red ones denote the initial positions of the spacecraft and the blue ones denote the final positions of the spacecraft. Whenever the case of a five-spacecraft formation reconfiguration or the case of a six-spacecraft formation reconfiguration is obtained from the adaptive surrogate model optimization, the relative distance of each spacecraft from the initial position to the final position is small. This means that the reconfiguration results from the adaptive surrogate model optimization satisfy the goal of minimizing fuel consumption.

Table 4 The specific final positions of the optimal spacecraft reconfiguration in local coordinates

\begin{tabular}{|c|c|c|c|c|c|c|c|}
\hline Reconfiguration cases & Position & $\# 1$ & $\# 2$ & \#3 & \#4 & $\# 5$ & \#6 \\
\hline \multirow{2}{*}{5 spacecraft } & $\mathrm{X}(\mathrm{m})$ & 617.285 & 254.015 & -333.771 & -333.771 & 254.015 & $\overline{---}$ \\
\hline & $\mathrm{Y}(\mathrm{m})$ & 0.000 & 500.000 & 309.017 & -309.017 & -500.000 & --- \\
\hline \multirow{2}{*}{6 spacecraft } & $\mathrm{X}(\mathrm{m})$ & -462.132 & -348.320 & 197.558 & 415.214 & 526.958 & 131.093 \\
\hline & $\mathrm{Y}(\mathrm{m})$ & 279.864 & -351.757 & -472.159 & -199.994 & 130.099 & 524.776 \\
\hline
\end{tabular}



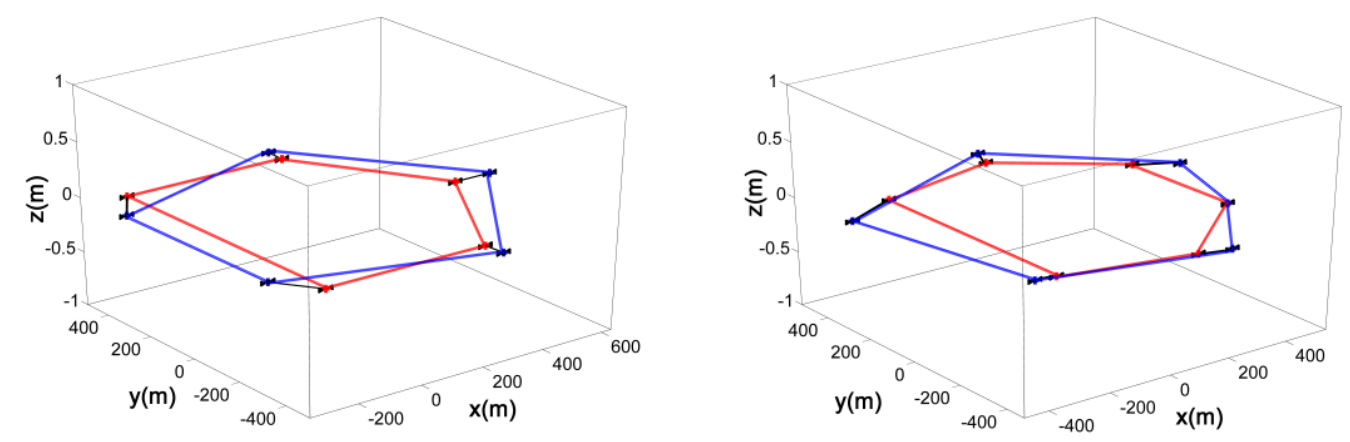

Fig. 4. Different cases of spacecraft formation reconfiguration: (a) five spacecraft; (b) six spacecraft

From the above discussions, we can obtain reasonable results for fast spacecraft formation reconfiguration through adaptive surrogate model optimization. Next, the optimality of the adaptive surrogate model optimization should be explained. Because the present adaptive surrogate model is a function of three input variables, i.e., the barycentric coordinates $x_{c}$ and $y_{c}$ and the rotation angle $\theta$, it cannot be intuitively and comfortably expressed with figures. To reduce the number of input variables and generate the figures for the adaptive surrogate model, the rotation angle $\theta$ of the final formation is fixed to be the optimal values of the adaptive surrogate model. Therefore, the input variables of the adaptive surrogate model are the barycentric coordinates $x_{c}$ and $y_{c}$, and the output variable of the adaptive surrogate model is the velocity increment $\Delta v$. It is easy to express and show the figures of the adaptive surrogate model. Figs. $5 \sim 8$ give the preliminary/final results of the adaptive surrogate model with a fixed rotation angle, and the optimum point is emphasized with a circle. The preliminary results of Fig. 5 and Fig. 7 are directly obtained from the first step of the adaptive surrogate model, according to the flow chart for generating the adaptive surrogate model. The final results of Fig. 6 and Fig. 8 are obtained from the second step of the adaptive surrogate model, according to the flow chart for generating the adaptive surrogate model.

As seen in Figs. 5 8, there are obvious difference between the adaptive surrogate model with five spacecraft and the adaptive surrogate model with six spacecraft. Furthermore, the RBF and the Kriging show little difference in the case of the five-spacecraft formation reconfiguration and show a large difference in the case of the six-spacecraft formation reconfiguration. However, either RBF or Kriging can find the optimal results, and these optimal results obtained from the RBF and the Kriging surrogate models are almost the same near the optimum point. The main reason for the difference between the 
RBF and the Kriging is the low density of the samples. These samples are far from the optimum point, and the global accuracy is not good in the region of the optimum point. Therefore, the optimality of the present adaptive surrogate model, i.e., the RBF and the Kriging, can be guaranteed in the process of fast spacecraft formation reconfiguration.

(a)

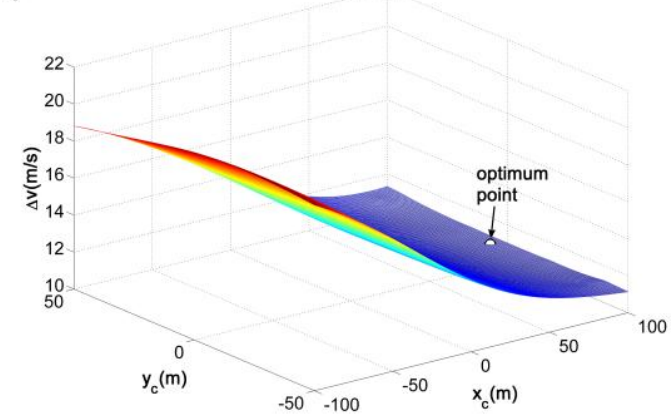

(b)

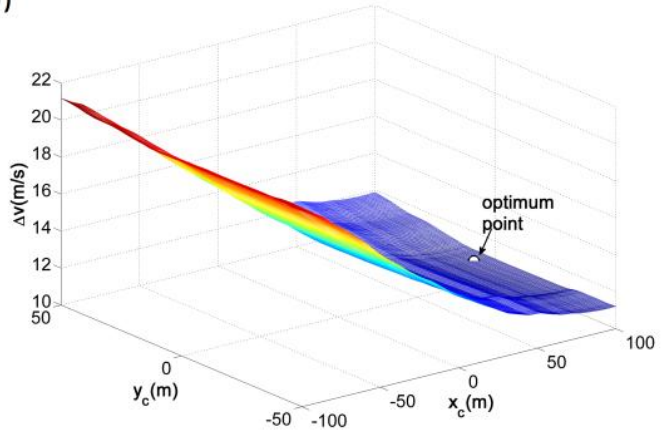

Fig. 5. The preliminary results of the SBO model with a fixed rotation angle (a) RBF results; (b)

Kriging results (five spacecraft in formation reconfiguration)

(a)

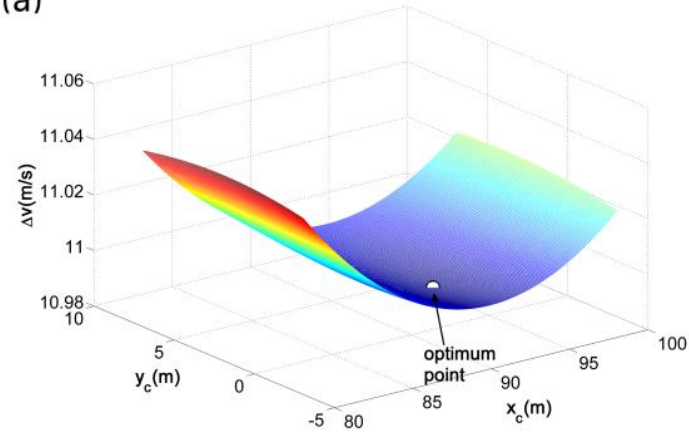

(b)

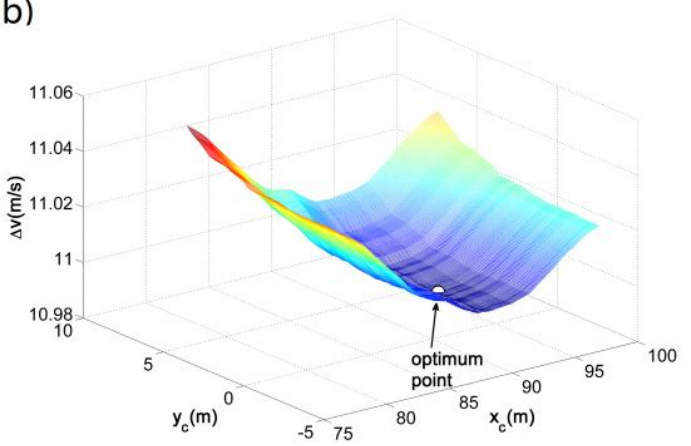

Fig. 6. The final results of the SBO model with a fixed rotation angle (a) RBF results; (b) Kriging results (five spacecraft in formation reconfiguration)

(a)

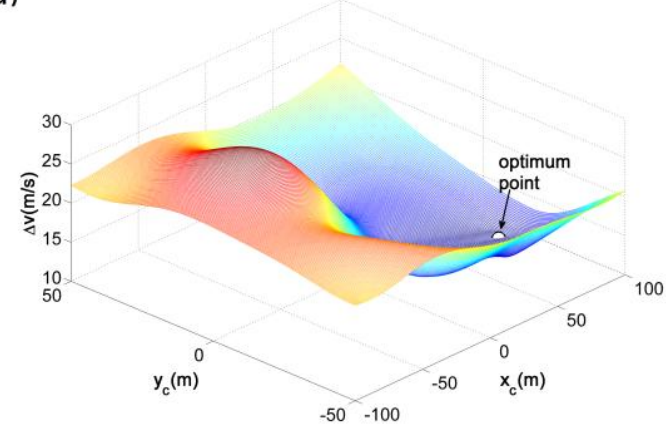

(b)

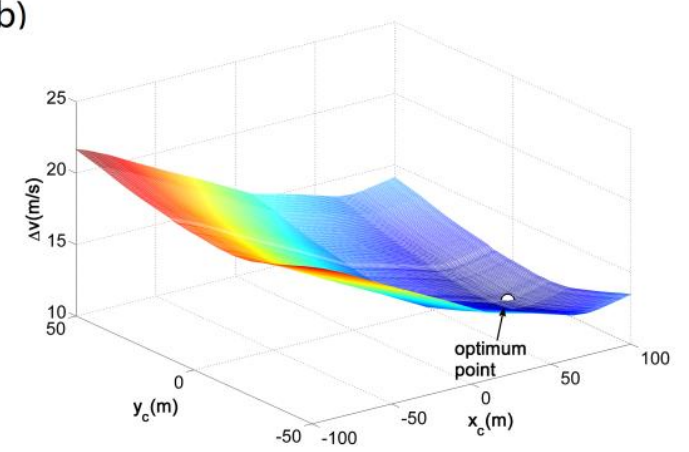

Fig. 7. The preliminary results of the SBO model with a fixed rotation angle (a) RBF results; (b) Kriging results (six spacecraft in formation reconfiguration) 
(a)

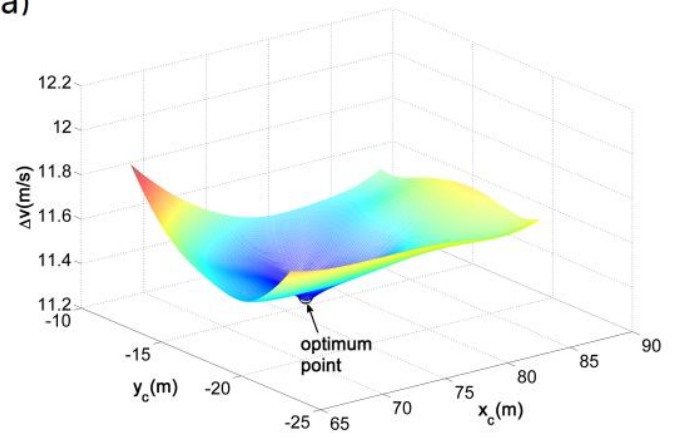

(b)

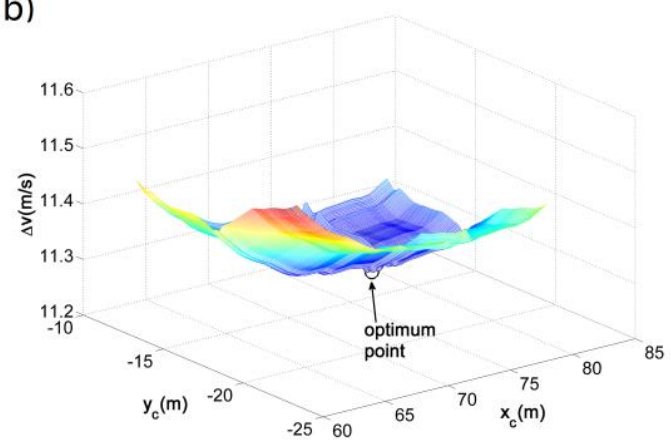

Fig. 8. The final results of the SBO model with a fixed rotation angle (a) RBF results; (b)

Kriging results (six spacecraft in formation reconfiguration)

In addition to the above qualitative discussions regarding the optimality of the adaptive surrogate model optimization, the accuracy of the adaptive surrogate model optimization can play a significant role in fast spacecraft formation reconfiguration. Tables 5 6 and Figs. 9 10 give the accuracy of the preliminary and final adaptive surrogate model results for different cases of formation reconfiguration. The relative error (RE) and absolute error (AE) of the input variables of the adaptive surrogate model $\theta$, $x_{c}$ and $y_{c}$ and the output variable of the adaptive surrogate model $\Delta v$, which are computed from the RBF and the Kriging surrogate model, are also given in Tables 5 6 and Figs. 9 10. The results from the ACO-based direct parameter optimization model are taken as reference solutions.

With the exception of one result in Table 5, we can see that the preliminary relative errors of the input/output variables obtained from both the RBF and the Kriging adaptive surrogate models are less than $10 \%$. Similar results can be found in Table 6 . The RBF and the Kriging adaptive surrogate models can give a higher accuracy, and most of the final relative errors of the input/output variables are less than 5\%. These results show that the final relative errors are smaller than the preliminary relative errors, and the adaptive strategy has increased the accuracy of the original surrogate models. Taking an extreme case as an example, the maximum relative error of the final result from the Kriging surrogate model is $8.0899 \%$ in Table 6 . The maximum absolute error value is $1.1874 \mathrm{~m}$ for input variable $y_{c}$. However, the changing range of input variable $y_{c}$ is from $-50 \mathrm{~m}$ to $50 \mathrm{~m}$. Therefore, the maximum absolute error value of $1.1874 \mathrm{~m}$ compared with the changing range of $100 \mathrm{~m}$ is also a small value.

From Tables 5 6 and Figs. 9 10, we can also find that when the number of spacecraft in the 
formation is odd, the accuracy of the adaptive surrogate model is better than for the spacecraft formation with an even number of spacecraft. From Figs. 5 8, we can also see that the surrogate model for an even number of spacecraft is more complex than that for an odd number of spacecraft. The main reason is that the optimal geometric shape of the spacecraft formation is an equilateral polygon for an odd number of spacecraft, according to the results of inner-loop optimization. However, the formation with an even number of spacecraft does not have such nice properties. Furthermore, the symmetry of the equilateral polygon can be employed to reduce the range of the rotation angle $\theta$. As a result, the accuracy of the adaptive surrogate model with an odd number of spacecraft in the formation is better than that for the spacecraft formation with an even number of spacecraft. In summary, either an odd or an even number of spacecraft from the adaptive surrogate model can satisfy the accuracy requirement for fast spacecraft formation reconfiguration.

Table 5 The accuracy of the preliminary results of the SBO model with different formation reconfigurations

\begin{tabular}{llllll}
\hline Reconfiguration cases & Methods & $\theta(\mathrm{rad})$ & $x_{c}(\mathrm{~m})$ & $y_{c}(\mathrm{~m})$ & $\Delta v(\mathrm{~m} / \mathrm{s})$ \\
\hline & ACO & 0 & 91.5542 & 0 & 10.9921 \\
& Kriging & 0 & 89.9998 & 2.4384 & 10.9867 \\
5 spacecraft & RE(\%) & --- & $\mathbf{1 . 6 9 7 8}$ & --- & $\mathbf{0 . 0 4 9 1}$ \\
& RBF & 0 & 92.0531 & 1.9386 & 10.9928 \\
& RE(\%) & --- & $\mathbf{0 . 5 4 4 9}$ & --- & $\mathbf{0 . 0 0 6 4}$ \\
& ACO & 2.6411 & 76.3915 & -14.6776 & 11.2630 \\
& Kriging & 2.6448 & 72.8574 & -16.1089 & 11.2440 \\
& RE(\%) & $\mathbf{0 . 1 4 0 1}$ & $\mathbf{4 . 6 2 6 3}$ & $\mathbf{9 . 7 5 1 6}$ & $\mathbf{0 . 1 6 8 7}$ \\
& RBF & 2.6410 & 77.2913 & -16.3202 & 11.3019 \\
& RE(\%) & $\mathbf{0 . 0 0 3 8}$ & $\mathbf{1 . 1 7 7 9}$ & $\mathbf{1 1 . 1 9 1 2}$ & $\mathbf{0 . 3 4 5 4}$ \\
\hline
\end{tabular}

Table 6 The accuracy of the final results of the SBO model with different formation reconfigurations

\begin{tabular}{llllll}
\hline Reconfiguration cases & Methods & $\theta(\mathrm{rad})$ & $x_{c}(\mathrm{~m})$ & $y_{c}(\mathrm{~m})$ & $\Delta v(\mathrm{~m} / \mathrm{s})$ \\
\hline \multirow{2}{*}{5 spacecraft } & ACO & 0 & 91.5542 & 0 & 10.9921 \\
& Kriging & 0 & 91.1771 & 0.5418 & 10.9941 \\
& RE(\%) & --- & $\mathbf{0 . 4 1 1 9}$ & --- & $\mathbf{0 . 0 1 8 2}$ \\
\hline
\end{tabular}




\begin{tabular}{llllll}
\hline & RBF & 0 & 91.5574 & 0.0287 & 10.9944 \\
& RE(\%) & $\mathbf{0}$ & $\mathbf{0 . 0 0 3 5}$ & --- & $\mathbf{0 . 0 2 0 9}$ \\
& ACO & 2.6411 & 76.3915 & -14.6776 & 11.2630 \\
& Kriging & 2.6457 & 75.6750 & -15.8650 & 11.2678 \\
6 spacecraft & RE(\%) & $\mathbf{0 . 1 7 4 2}$ & $\mathbf{0 . 9 3 7 9}$ & $\mathbf{8 . 0 8 9 9}$ & $\mathbf{0 . 0 4 2 6}$ \\
& RBF & 2.6427 & 76.0866 & -15.3817 & 11.2603 \\
& RE(\%) & $\mathbf{0 . 0 6 0 6}$ & $\mathbf{0 . 3 9 9 1}$ & $\mathbf{4 . 7 9 7 1}$ & $\mathbf{0 . 0 2 4 0}$ \\
\hline
\end{tabular}
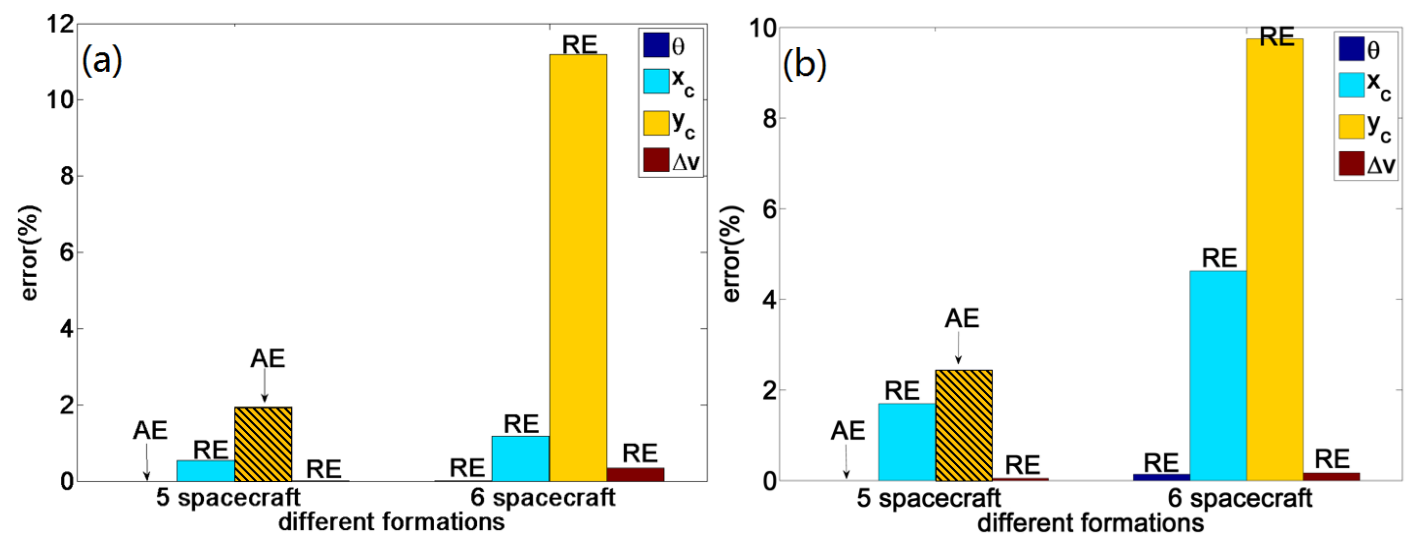

Fig. 9 The relative/absolute errors of the preliminary results of the SBO model (a) RBF; (b) Kriging
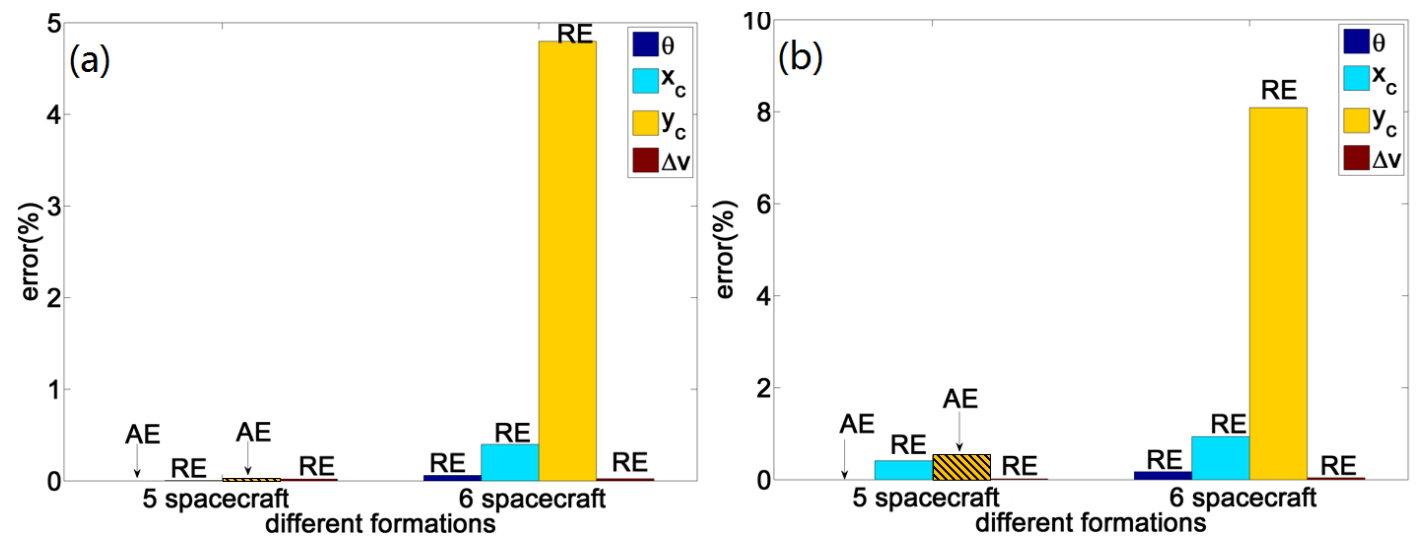

Fig. 10 The relative/absolute errors of the final results of the SBO model (a) RBF; (b) Kriging

\section{2. $\quad$ Efficiency}

In addition to accuracy, the efficiency of the adaptive surrogate model is another important factor in the practical implementation of fast spacecraft formation reconfiguration. Therefore, direct parameter optimization without using the adaptive surrogate model is also employed for comparison. As is well known, intelligent algorithms and gradient-based optimization algorithms are two general 
types of optimization methods. Intelligent algorithms have the capability of obtaining the global optimum result at the cost of an enormous amount of computation. The gradient-based optimization algorithm has a smaller computational cost but has a poor ability to obtain the global optimum result. However, the surrogate model-based optimization is an approximate method that has a good ability to obtain the global optimum result. Meanwhile, its computational cost is much lower than that of intelligent algorithms. To compare the efficiency of the different methods, two computational cost indicators, i.e., the computational time of implementing the programs and the number of solved nonlinear optimal control problems, are obtained for all the methods. Therefore, Fig. 11 shows the computational time of the ACO method without the surrogate model and the RBF and the Kriging surrogate models for different cases. Table 7 gives the number of evaluated points, i.e., the number of solved nonlinear optimal control problems, for the RBF, Kriging and ACO.

From Fig. 11, the computational time of the ACO method is much larger than that of the RBF and Kriging surrogate models. The computational time of the RBF and Kriging surrogate models is approximately $5 \%$ that of the ACO method. Therefore, the efficiency of the adaptive surrogate model is an obvious advantage. Actually, the influence factor of computational time can be found from Table 7. The total number of evaluated points for both the RBF and the Kriging surrogate models is much smaller than the number of evaluated points for ACO-based direct parameter optimization. The greater the number of evaluated points that exist in the process of optimization is, the more nonlinear optimal control problems need to be solved. Therefore, most of the computational time for ACO-based direct parameter optimization has been spent on solving the nonlinear optimal control problems.

In fact, the computational times for both the RBF and the Kriging methods are mostly spent on constructing the adaptive surrogate models. In the process of generating the adaptive surrogate models, only a few hundreds of evaluated points need to be implemented. Once the adaptive surrogate models are obtained, both the RBF and Kriging methods give the explicit relationships between the input variables $\theta, x_{c}$ and $y_{c}$ and the output variable $\Delta v$. Meanwhile, based on the explicit relationships, the optimal results of parameter optimization can be easily obtained by employing intelligent algorithms or gradient-based optimization algorithms. Moreover, no nonlinear optimal control problem should be solved for the adaptive surrogate models in the process of parameter optimization. Therefore, the adaptive surrogate model-based parameter optimization has almost the same accuracy as the direct parameter optimization, but it has a high-efficiency optimization. 


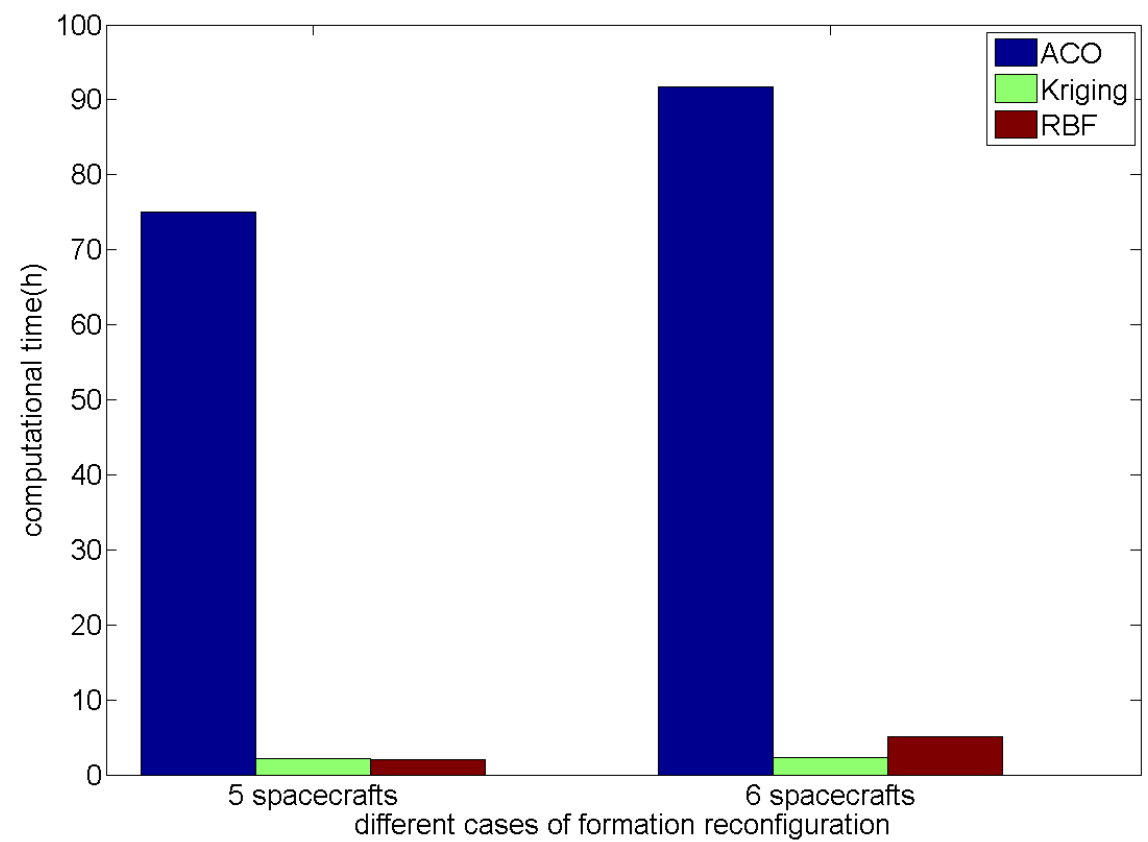

Fig. 11 Comparisons of the ACO, RBF and Kriging methods on computational time for different cases

Table 7 The number of evaluated points with different reconfiguration cases (1 hour reconfiguration)

\begin{tabular}{lllll}
\hline Reconfiguration cases & Design phases & Kriging & RBF & ACO \\
\hline \multirow{3}{*}{5 spacecraft } & First step & 195 & 195 & --- \\
& Second step & 60 & 60 & --- \\
& Total number & $\mathbf{2 5 5}$ & $\mathbf{2 5 5}$ & $\mathbf{1 0 0 0 0}$ \\
& First step & 165 & 435 & --- \\
6 spacecraft & Second step & 60 & 75 & --- \\
& Total number & $\mathbf{2 2 5}$ & $\mathbf{5 1 0}$ & $\mathbf{1 0 0 0 0}$ \\
\hline
\end{tabular}

\section{3.}

Robustness

The robustness of the proposed adaptive surrogate model optimization with respect to parameters will be studied in this subsection. There are many parameters that can affect the performance of the adaptive surrogate model optimization. One key factor of these important parameters is the reconfiguration time of the spacecraft formation. It is obvious that different reconfiguration times will require different fuel consumptions. In this part, the case of formation reconfiguration with five 
spacecraft is considered for different reconfiguration times. The other parameters of the spacecraft formation reconfiguration are the same as those in the previous parts. Tables $8 \sim 10$ give the accuracy of the final results of the RBF and Kriging adaptive surrogate model optimization for five spacecraft within the reconfiguration times $0.5,2$ and 4 hours, respectively. Fig. 12 shows the relative/absolute errors of the final results for both Kriging and RBF methods with different reconfiguration times.

From Tables 6, 8, 9 and 10 and Figure 12, we can see that the accuracy of the final results for both the Kriging and RBF methods satisfies the requirements for fast spacecraft formation reconfiguration. The accuracy of the final results is not strongly influenced by the different reconfiguration times. The relative error of the final results for the RBF method is less than $0.08 \%$ and for the Kriging method is less than $1.4 \%$. Therefore, the RBF-based adaptive surrogate model optimization has a better accuracy than the Kriging-based adaptive surrogate model optimization, and they both prove the robustness of the proposed adaptive surrogate model optimization.

Table 8 The accuracy of the final results of the SBO model for five spacecraft with 0.5 hours

\begin{tabular}{lllll}
\hline Methods and errors & $\theta(\mathrm{rad})$ & $x_{c}(\mathrm{~m})$ & $y_{c}(\mathrm{~m})$ & $\Delta v(\mathrm{~m} / \mathrm{s})$ \\
\hline ACO & 0 & 91.5358 & 0.0280 & 21.7528 \\
Kriging & 0.0004 & 91.3201 & -0.2174 & 21.7530 \\
RE(\%) & --- & $\mathbf{0 . 2 3 5 6}$ & --- & $\mathbf{0 . 0 0 0 9}$ \\
RBF & 0 & 91.5540 & 0.0449 & 21.7531 \\
$\mathbf{R E}(\%)$ & --- & $\mathbf{0 . 0 1 9 9}$ & --- & $\mathbf{0 . 0 0 1 4}$ \\
\hline
\end{tabular}

Table 9 The accuracy of the final results of the SBO model for five spacecraft with 2 hours

\begin{tabular}{lllll}
\hline Methods and errors & $\theta(\mathrm{rad})$ & $x_{c}(\mathrm{~m})$ & $y_{c}(\mathrm{~m})$ & $\Delta v(\mathrm{~m} / \mathrm{s})$ \\
\hline ACO & 0 & 91.5539 & -0.0014 & 5.4618 \\
Kriging & 0.0013 & 91.0345 & -1.3539 & 5.4621 \\
$\mathbf{R E}(\%)$ & --- & $\mathbf{0 . 5 6 7 3}$ &.-- & $\mathbf{0 . 0 0 5 5}$ \\
$\operatorname{RBF}$ & 0 & 91.5544 & 0.0211 & 5.4621 \\
$\mathbf{R E}(\%)$ & --- & $\mathbf{0 . 0 0 0 6}$ & --- & $\mathbf{0 . 0 0 5 5}$ \\
\hline
\end{tabular}

Table 10 The accuracy of the final results of the SBO model for five spacecraft with 4 hours 


\begin{tabular}{lllll}
\hline Methods and errors & $\theta(\mathrm{rad})$ & $x_{c}(\mathrm{~m})$ & $y_{c}(\mathrm{~m})$ & $\Delta v(\mathrm{~m} / \mathrm{s})$ \\
\hline ACO & 0 & 91.5331 & -0.0003 & 2.6690 \\
Kriging & 0.0009 & 91.3449 & -0.5172 & 2.6690 \\
RE $(\%)$ & --- & $\mathbf{0 . 2 0 5 6}$ & --- & $\mathbf{0 . 0 0 0 0}$ \\
RBF & 0 & 91.5559 & 0.0102 & 2.6690 \\
$\mathbf{R E}(\%)$ & --- & $\mathbf{0 . 0 2 4 9}$ & --- & $\mathbf{0 . 0 0 0 0}$ \\
\hline
\end{tabular}
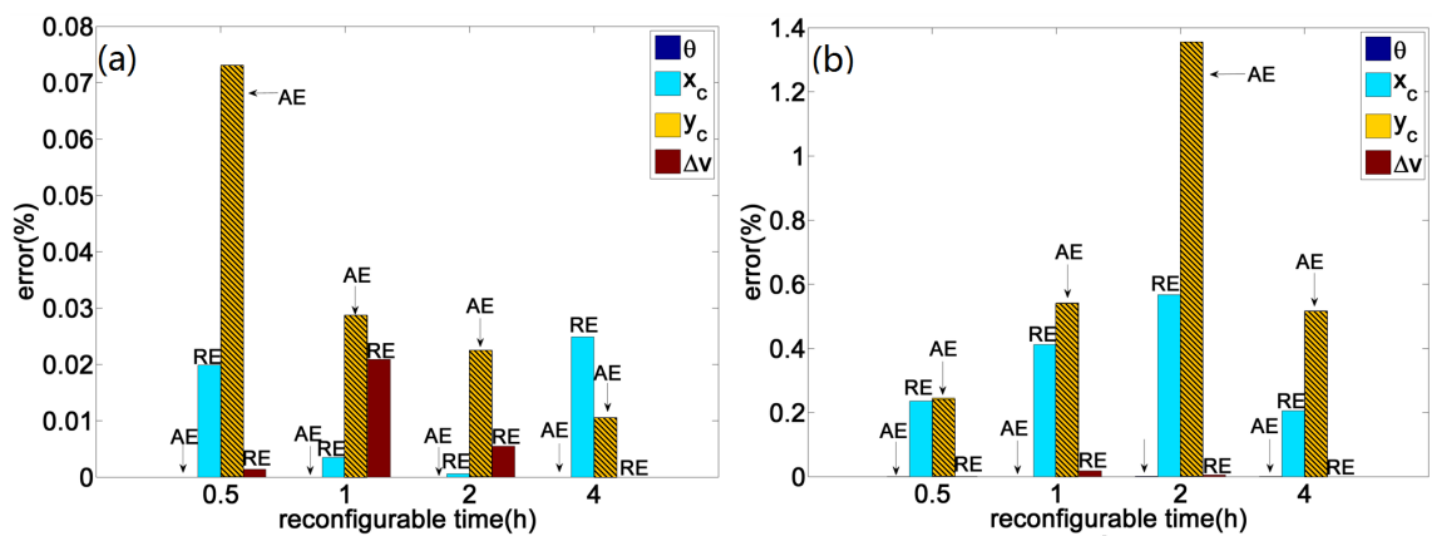

Fig. 12 The relative/absolute errors of the final results of the SBO model for different reconfiguration times (a) RBF; (b) Kriging

In addition to the effect of the reconfiguration time on accuracy, the parameter influence of the reconfiguration time on computational cost is also studied. Table 11 shows the comparison of the number of evaluated points for five spacecraft with different reconfiguration times. Fig. 13 shows the comparison of the computational time for the ACO, RBF and Kriging methods with different reconfiguration times. It is obvious that the number of evaluated points or computational time for the RBF and Kriging methods is much smaller than that for ACO-based direct parameter optimization. Furthermore, changing the parameter of reconfiguration time has little influence on efficiency. Moreover, the computational cost of the RBF-based adaptive surrogate model optimization is a little lower than that of the Kriging-based adaptive surrogate model optimization.

Table 11 The number of evaluated points for five spacecraft with different reconfiguration times

\begin{tabular}{llllc}
\hline Times (hour) & Design phases & Kriging & RBF & ACO \\
\hline
\end{tabular}




\begin{tabular}{lllll}
\hline & First step & 225 & 165 & --- \\
0.5 & Second step & 180 & 75 & --- \\
Total number & $\mathbf{4 0 5}$ & $\mathbf{2 4 0}$ & $\mathbf{1 0 0 0 0}$ \\
& First step & 195 & 195 & --- \\
& Second step & 60 & 60 & --- \\
& Total number & $\mathbf{2 5 5}$ & $\mathbf{2 5 5}$ & $\mathbf{1 0 0 0 0}$ \\
& First step & 195 & 135 & --- \\
& Second step & 75 & 60 & --- \\
& Total number & $\mathbf{2 7 0}$ & $\mathbf{1 9 5}$ & $\mathbf{1 0 0 0 0}$ \\
& First step & 150 & 150 & --- \\
& Second step & 210 & 60 & --- \\
& Total number & $\mathbf{3 6 0}$ & $\mathbf{2 1 0}$ & $\mathbf{1 0 0 0 0}$ \\
\hline
\end{tabular}

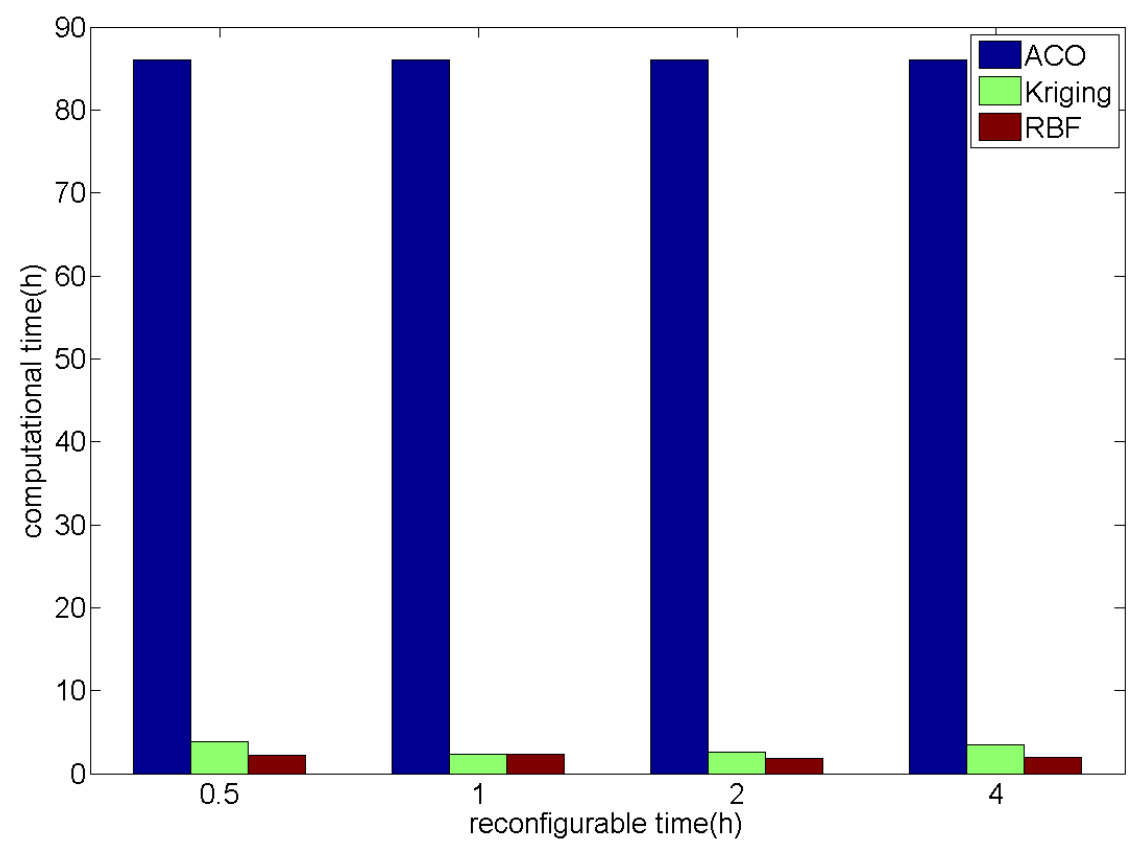

Fig. 13 Comparisons of the ACO, RBF and Kriging methods on computational time for different reconfiguration times

\section{Conclusions}

An adaptive surrogate model, including RBF and Kriging surrogate models, is proposed to solve 
the multi-layer optimization and obtain the fast optimal transfer paths of spacecraft formation reconfiguration on libration point orbits. Compared with direct optimization based on the original multi-layer optimization model, the optimality of the present adaptive surrogate model, i.e., the RBF and the Kriging, can be guaranteed in the processing of fast spacecraft formation reconfiguration. Meanwhile, the RBF and the Kriging adaptive surrogate models can give a higher accuracy; most of the final relative errors of the input/output variables are less than $5 \%$. The computational time of the RBF and Kriging surrogate models is only approximately $5 \%$ that of the ACO method based on the original multi-layer optimization model. Therefore, the efficiency of the adaptive surrogate model has obvious advantages. Furthermore, changing the parameter of spacecraft formation reconfiguration time has no obvious influence on the accuracy and efficiency. Therefore, the present adaptive surrogate model has good robustness.

\section{Acknowledgments}

The authors are grateful for the financial support of the National Science Foundation of China (11472069, 11432010); the Project Funded by China Postdoctoral Science Foundation (2014M550155; 2015T80245); the State Key Laboratory of Mechanics and Control of Mechanical Structures (MCMS-0114G02); and the Dalian Science and Technology Project (2015A11GX037).

\section{References}

[1].H.J. Peng, C.F. Yang, Y.P. Li, S Zhang, B.S. Chen, Surrogate-based parameter optimization and optimal control for optimal trajectory of Halo orbit rendezvous, Aerospace Science and Technology 26(9) (2013) 176-184.

[2].H.J. Peng, B.S. Chen, Z.G. Wu, Multi-objective transfer to libration-point orbits via the mixed low-thrust and invariant-manifold approach, Nonlinear Dynamics 77(1-2) (2014) 321-338.

[3].H.J. Peng, Q. Gao, Z.G. Wu, W.X. Zhong, Optimal guidance based on receding horizon control for low-thrust transfer to libration point orbits. Advances in Space Research 51(11) (2013) 2093-2111.

[4].S.N. Wu, Z.G. Wu, G. Radice, R. Wang, Adaptive control for spacecraft relative translation with parametric uncertainty, Aerospace Science and Technology 31(1) (2013) 53-58.

[5].L.D. Millard, K.C. Howell, Optimal reconfiguration maneuvers for spacecraft imaging arrays in 
multi-body regimes, Acta Astronautica 63(11-12) (2008) 1283-1298.

[6].L. Garcia-Taberner, J.J. Masdemont, FEFF methodology for spacecraft formations reconfiguration in the vicinity of libration points, Acta Astronautica 67(7-8) (2010) 810-817.

[7].C. Park, J.H. Yang, D.J. Scheeres, Optimal formation reconfigurations subject to Hill three-body dynamics, Journal of Guidance, Control, and Dynamics 37(2) (2014) 700-704.

[8].C. Sultan, S. Seereram, R.K. Mehra, Deep space formation flying spacecraft path planning. The International Journal of Robotics Research 26(4) (2007) 405-430.

[9].L.F. Peñin, J. Araújo, N. Ávila, Design and evaluation of optimal reconfiguration maneuvers for separated space interferometry. Acta Astronautica 57(2-8) (2005) 330-340.

[10].B. Açıkmese, D.P. Scharf, E.A. Murray, F.Y. Scharf, A convex guidance algorithm for formation reconfiguration. In: AIAA Guidance, Navigation, and Control Conference and Exhibit, Colorado, USA. AIAA 2006-6070 (2006).

[11].J. Nocedal, S.J. Wright, Numerical Optimization, 2nd edition, Springer (2006).

[12].M. Dorigo, T. Stutzle, Ant Colony Optimization, MIT Press (2004).

[13].S. Vakili, M.S. Gadala, Low cost surrogate model based evolutionary optimization solvers for inverse heat conduction problem, International Journal of Heat and Mass Transfer 56 (11) (2013) 263-273.

[14].Y.V. Pehlivanoglu, B. Yagiz, Aerodynamic design prediction using surrogate-based modeling in genetic algorithm architecture, Aerospace Science and Technology 23(1) (2012) 479-491.

[15].A.I J. Forrester, A.J. Keane, Recent advances in surrogate-based optimization, Progress in Aerospace Sciences, 45(1-3) (2009) 50-79.

[16].N.V. Queipo, R.T. Haftka, W. Shyy, T. Goel, R. Vaidyanathan, P.K. Tucker, Surrogate-based analysis and optimization, Progress in Aerospace Sciences 41 (1) (2005) 1-28.

[17].D.L. Richardson, Analytic construction of periodic orbits about the collinear points, Celestial Mechanics and Dynamical Astronomy 22 (13) (1980) 241-253.

[18].M. Popescu, V. Cardoş, The domain of initial conditions for the class of three-dimensional halo periodical orbits, Acta Astronautica 36 (4) (1995) 193-196.

[19].Y. Zhang, J. Chen, L.C. Shen, Real-time trajectory planning for UCAV air-to-surface attack using inverse dynamics optimization method and receding horizon control, Chinese Journal of Aeronautics 26 (4) (2013) 1038-1056. 
[20].H.J. Peng, X. Jiang, B.S. Chen, Optimal nonlinear feedback control of spacecraft rendezvous with finite low thrust between libration orbits, Nonlinear Dynamics 76 (22) (2014) 1611-1632.

[21].H.J. Peng, Q. Gao, Z.G. Wu, W.X. Zhong, Symplectic adaptive algorithm for solving nonlinear two-point boundary value problems in astrodynamics, Celestial Mechanics and Dynamical Astronomy 110 (24) (2011) 319-342.

[22].J.E. Kulkarni, M.E. Campbell, G.E. Dullerud, Stabilization of spacecraft flight in halo orbits: an HN approach, IEEE Transactions on Control Systems Technology 14 (3) (2006) 572-578.

[23].R. Menasri, A. Nakib, B. Daachi, H. Oulhadj, P. Siarry, A trajectory planning of redundant manipulators based on bilevel optimization, Applied Mathematics and Computation 250 (2015) 934-947.

[24].L. Garcia-Taberner, J.J. Masdemont, FEFF methodology for spacecraft formations reconfiguration in the vicinity of libration points, Acta Astronautica 67(7-8) (2010) 810-817.

[25].H. Huang, Y. Zhuang, Optimal satellite formation reconfiguration using co-evolutionary particle swarm optimization in deep space, Acta Astronautica 113 (2015) 149-163.

[26].P. Hao, B. Wang, G. Li, Surrogate-based optimum design for stiffened shells with adaptive sampling, AIAA Journal, 50(11) (2012) 2389-2407.

[27].K.T. Fang, D.K.J. Lin, Uniform experimental designs and their applications in industry, Handbook of Statistics, (2003) 131-170.

[28].S.E. Gano, H. Kim, D.E. Brown, Comparison of three surrogate modeling techniques: Datascape ${ }^{\circledR}$, Kriging, and Second order regression, Proceedings of the AIAA/ISSMO Multidisciplinary Analysis and Optimization Conference, No. AIAA 2006-7048, Portsmouth, Virginia, September 2006.

[29].M. L. Stein, Interpolation of Spatial Data: Some Theory for Kriging, Springer-Verlag, 1999.

[30].Y. Jin, A comprehensive survey of fitness approximation in evolutionary computation, Soft Computing 9 (1) (2005) 3-12. 